\title{
AIP
}

\section{Structure and transport properties of polymer grafted nanoparticles}

Sushmit Goyal and Fernando A. Escobedo

Citation: J. Chem. Phys. 135, 184902 (2011); doi: 10.1063/1.3657831

View online: http://dx.doi.org/10.1063/1.3657831

View Table of Contents: http://jcp.aip.org/resource/1/JCPSA6/v135/i18

Published by the AIP Publishing LLC.

\section{Additional information on J. Chem. Phys.}

Journal Homepage: http://jcp.aip.org/

Journal Information: http://jcp.aip.org/about/about_the_journal

Top downloads: http://jcp.aip.org/features/most_downloaded

Information for Authors: http://jcp.aip.org/authors

\section{ADVERTISEMENT}

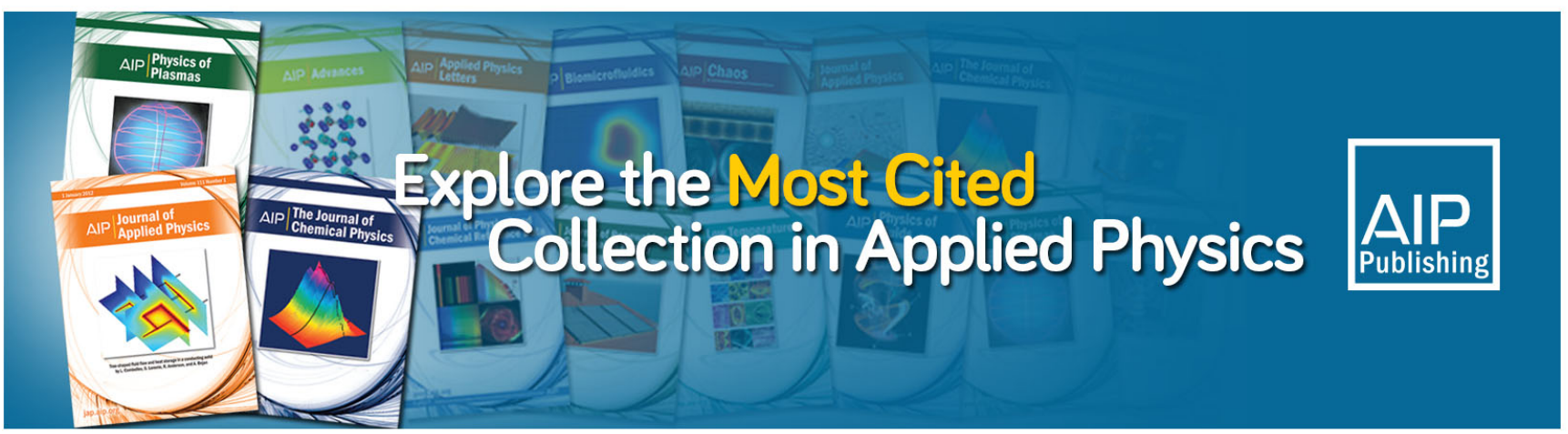




\title{
Structure and transport properties of polymer grafted nanoparticles
}

\author{
Sushmit Goyal and Fernando A. Escobedoa) \\ Department of Chemical and Biomolecular Engineering, Cornell University, Ithaca, New York 14850, USA
}

(Received 26 August 2011; accepted 13 October 2011; published online 9 November 2011)

\begin{abstract}
We perform molecular dynamics simulations on a bead-spring model of pure polymer grafted nanoparticles (PGNs) and of a blend of PGNs with a polymer melt to investigate the correlation between PGN design parameters (such as particle core concentration, polymer grafting density, and polymer length) and properties, such as microstructure, particle mobility, and viscous response. Constant strain-rate simulations were carried out to calculate viscosities and a constant-stress ensemble was used to calculate yield stresses. The PGN systems are found to have less structural order, lower viscosity, and faster diffusivity with increasing length of the grafted chains for a given core concentration or grafting density. Decreasing grafting density causes depletion effects associated with the chains leading to close contacts between some particle cores. All systems were found to shear thin, with the pure PGN systems shear thinning more than the blend; also, the pure systems exhibited a clear yielding behavior that was absent in the blend. Regarding the mechanism of shear thinning at the high shear rates examined, it was found that the shear-induced decrease of Brownian stresses and increase in chain alignment, both correlate with the reduction of viscosity in the system with the latter being more dominant. A coupling between Brownian stresses and chain alignment was also observed wherein the non-equilibrium particle distribution itself promotes chain alignment in the direction of shear. (C) 2011 American Institute of Physics. [doi:10.1063/1.3657831]
\end{abstract}

\section{INTRODUCTION}

Polymer nanocomposites have been a topic of interest in recent years as their unique properties have been exploited for applications such as desalination, $\mathrm{CO}_{2}$ capture, photovoltaics, and immersion lithography. ${ }^{1-4}$ Unlike colloids which tend to agglomerate irreversibly, suspensions of polymer grafted nanoparticles (PGNs) are stabilized by polymer-polymer steric interactions. Nanoscale organic hybrid materials, which are a class of such materials, consist of an inorganic nanoparticle core, functionalized with a corona of organic oligomers. These differ from common nanocomposites in which the tethered corona is the suspending medium for the cores, ${ }^{2-6}$ the grafted polymers tend to fill in the space between the inorganic cores to form the suspending fluid "phase". The hybrid nature of the suspension allows the fabrication of materials with tunable properties by varying parameters of both the organic polymers (such as molecular weight and grafting density) and the nanoparticle cores (such as chemistry, shape, and size). The properties exhibited by these composites vary from solids, stiff waxes, and gels for systems of high core content to solvent free fluids ${ }^{4-6}$ for systems of low core content.

Theoretical, experimental, and computational studies have been carried out to elucidate the transport properties of polymer grafted nanoparticles in a polymer matrix, ${ }^{1,7-11}$ a wetting regime was identified where the surrounding polymer matrix interpenetrates the grafted nanoparticles, effectively wetting and stabilizing them. Various theoretical and

\footnotetext{
a) Author to whom correspondence should be addressed. Electronic mail: fe13@cornell.edu.
}

computational studies have also been carried out on the structural effect of tethering chains, chain length, and tether location., ${ }^{5,9}$ For pure PGN systems, various experimental studies have been carried out to characterize the rheology of brush-type PGNs where the thickness of grafted polymers is comparable to the nanoparticle radius, finding a distinctive shear thinning behavior and a marked influence of polymer chemistry on viscosities. ${ }^{3}$ Molecular simulation studies of these systems have been initiated only very recently. ${ }^{12}$

Given the different ways in which a single PGN can be modified, molecular simulations can help elucidate how their bulk properties are affected (and can be optimized for specific applications), by changes in molecular design. To this end, we have applied molecular dynamics to get a better understanding of structure and transport properties of these systems. In this work we investigate the equilibrium and rheological properties of PGNs. The simulated systems were chosen to roughly mimic some experimental systems made of silica cores and PEO chains (having high grafting density and short chains ${ }^{2}$ ) and to isolate the contributions of core and corona on PGN's dynamics by varying the core volume fraction and polymer length. PGNs with varying core density and corona size were hence simulated wherein non-equilibrium methods were implemented to obtain both viscosities (by imposing a homogeneous steady state shear) and the yield stress. A non-Newtonian shear thinning behavior is observed in all cases with a lower shear thinning slope for PGNs in a blend as compared to pure PGNs. In Sec. II we introduce our model followed by the methodology used to calculate the system's properties (Sec. III). We then present and analyze our results in Sec. IV and give some concluding remarks in Sec. V. 


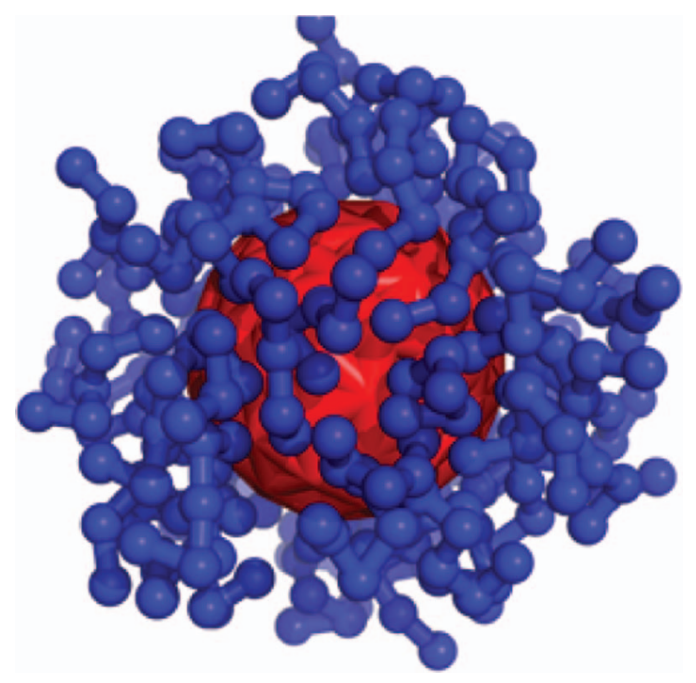

FIG. 1. Schematic of a typical PGN.

\section{MODEL}

Figure 1 represents a schematic of a single PGN particle. The nanoparticle component is modeled as a hollow spherical shell made of 80 Lennard Jones (LJ) beads forming the surface and a single bead in the center. All LJ beads have a diameter of $\sigma$ which gives the nanoparticle core an outer diameter of $D=6 \sigma$ (Fig. 2(a)). To fix the LJ beads on a spherical surface, each bead on the surface is attached to its neighboring beads and also to the center bead by harmonic bonds (Figs. 2(b) and 2(c)). These surface LJ beads are used as grafting points to permanently anchor polymers to the surface of the nanoparticle. Tethered polymers are freely jointed chains of $\mathrm{N}_{\mathrm{m}}$ beads. For the blend system (PGNs + polymer melt), the surrounding fluid has chains of $\mathrm{N}_{\mathrm{fp}}$ beads each and the ratio of number of tethered chain beads to number of free chain beads is given by $\mathrm{P}_{\mathrm{f}}$.

For computational efficiency, we coarse grained our system to reduce the number of particles required. We have not used the usual dissipative particle dynamics soft potential as it contains only repulsive interactions which would not capture non-ideal chain conformational behavior arising from attractive interactions and the non-crossability of chains. We have instead used a coarse grained chain model with Lennard Jones beads and stiff bonds noting that an $\mathrm{N}_{\mathrm{m}}$-bead oligomer could not only represent a chain with $\mathrm{N}_{\mathrm{m}}$ Kuhn segments but also approximate a bundle of a few oligomer chains. To roughly map LJ units into real units, a value of $\sigma \approx 1 \mathrm{~nm}$ can be estimated by mapping the core diameter to the smallest diameter of silica particles typically used experimentally., ${ }^{2,6}$

The grafting density (GD) gives what percentage of the maximum possible number of chains has been attached to the nanoparticle surface. It is calculated in our model as

$$
\mathrm{GD}=\frac{\text { Number of chains attached }}{\text { Number of surface beads }} \times 100 \text {. }
$$

When GD $<100$, grafting points are chosen randomly from the 80 surface beads so as to produce a relatively uniform (a)

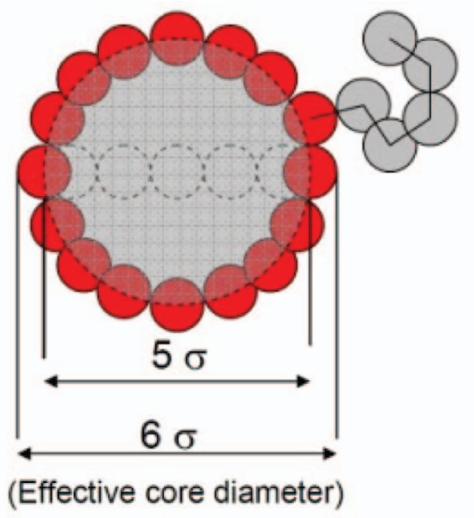

(b)

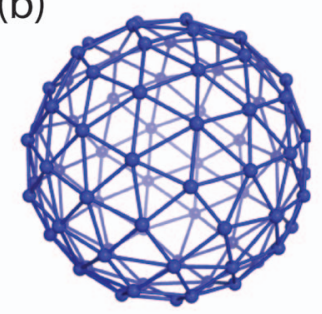

(c)

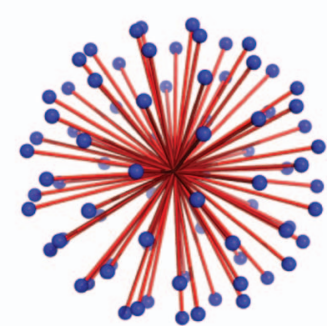

FIG. 2. Schematics of (a) approximate dimensions of PGNs, (b) surface beads and bonds joining neighboring particles without center bead, and (c) core structure showing center bead and bonds with surface beads.

coverage. The volume fraction of cores $\left(\phi_{c}\right)$ is calculated as

$$
\phi_{c}=\frac{\text { (number of cores) } \times \frac{\pi}{6} D^{3}}{\text { Volume of simulation box }} .
$$

Figures 3(a) and 3(b) show snapshots of some of the systems simulated and Fig. 3(c) and Table I summarize the key characteristics of the systems simulated in this study.

To identify the various simulated systems we use a three variable naming scheme where we represent each system using the formulae $\mathrm{N}_{\mathrm{m}}-\mathrm{GD}-\phi_{\mathrm{c}}$, which gives the chains length $\left(\mathrm{N}_{\mathrm{m}}\right)$, grafting density (GD), and core volume fraction $\left(\phi_{c}\right)$. For the case when we have simulated a blend, we use the formula $\mathrm{N}_{\mathrm{m}}-\mathrm{GD}-\phi_{\mathrm{c}}\left(\mathrm{N}_{\mathrm{fp}}-\mathrm{P}_{\mathrm{f}}\right)$, which specifies the needed additional parameters, namely, chain length $\left(\mathrm{N}_{\mathrm{fp}}\right)$ of free polymers and the volume ratio of free chains to total chains $\left(\mathrm{P}_{\mathrm{f}}\right)$.

\begin{tabular}{|c|c|c|c|c|c|c|}
\hline System & $\mathrm{N}_{\mathrm{m}}$ & GD & $\phi_{c}$ & Number of cores & & Box length, $\mathrm{L}_{\text {box }}$ \\
\hline $\mathrm{A}$ & 5 & 80 & 0.23 & 256 & & 50.41 \\
\hline A-100 & 5 & 100 & 0.19 & 256 & & 53.41 \\
\hline A-50 & 5 & 50 & 0.32 & 256 & & 45.10 \\
\hline B & 10 & 80 & 0.13 & 256 & & 61.25 \\
\hline B-50 & 10 & 50 & 0.19 & 256 & & 53.55 \\
\hline $\mathrm{C}$ & 20 & 40 & 0.13 & 108 & & 45.93 \\
\hline Blend & $\mathrm{N}_{\mathrm{m}}$ & $\mathrm{P}_{\mathrm{f}}$ & $\phi_{c}$ & Number of cores & $\mathrm{N}_{\mathrm{fp}}$ & $\mathrm{L}_{\text {box }}$ \\
\hline$\overline{\mathrm{D}}$ & 5 & 0.5 & 0.13 & 108 & 5 & 45.93 \\
\hline
\end{tabular}

TABLE I. Simulation parameters of the various systems studied. 
(a)

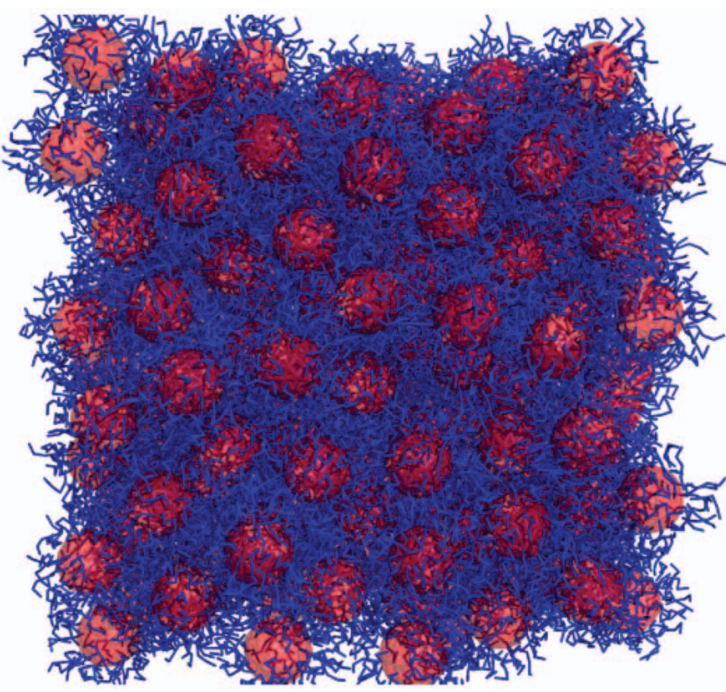

(b)

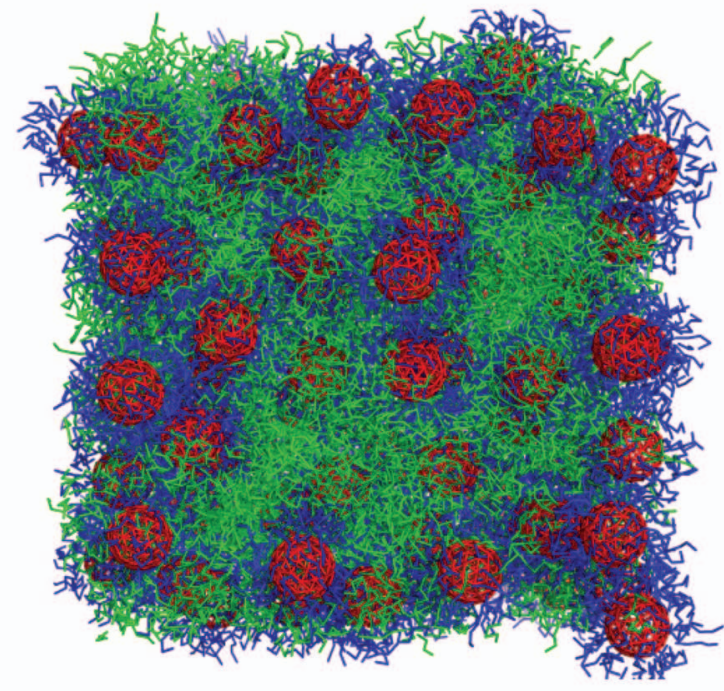

(c)

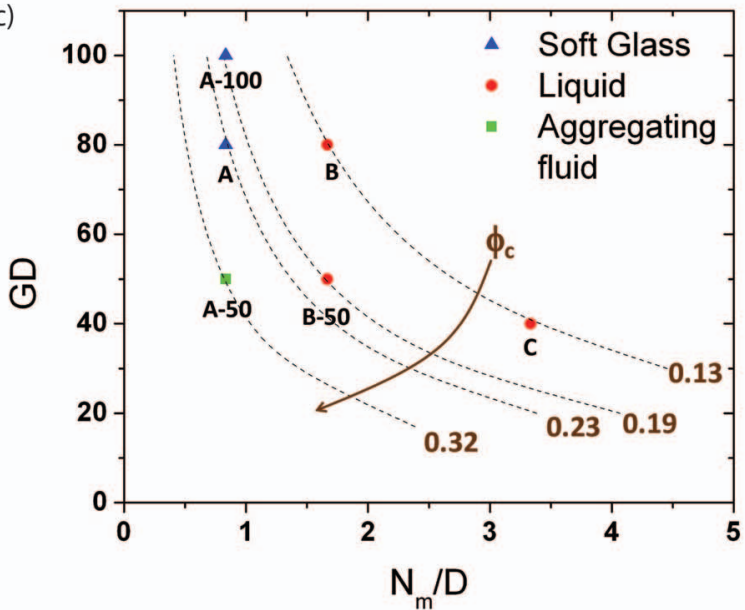

FIG. 3. Snapshots of sample simulated systems: (a) System A with $\phi_{c}$ $=0.23, \mathrm{~N}_{\mathrm{m}}=5$, and GD $=80 \%$; (b) System D, PGNs in blend with polymer with $\phi_{c}=0.13, \mathrm{~N}_{\mathrm{m}}=5$, and $\mathrm{N}_{\mathrm{fp}}=5$. (c) Summary of pure PGN systems explored in the GD vs $\mathrm{N}_{\mathrm{m}} / \mathrm{D}$ space with isolines shown for different $\phi_{c}$ values. The meaning of the symbol legends is described in Sec. IV.

The pair interaction potential used between non-bonded beads is the cut-shifted Lennard Jones force potential which gives a continuous potential and force, the potential is given as $^{13}$

$$
\begin{aligned}
& v^{s f}=\left\{\begin{array}{cc}
v-v_{\text {cutoff }}-\left(r-r_{c}\right)\left(\frac{d v}{d r}\right)_{r_{c}}, & r_{i j}<r_{c} \\
0, & r_{i j}>r_{c}
\end{array}\right\}, \\
& v=4 \varepsilon\left[\left(\frac{\sigma}{r}\right)^{12}-\left(\frac{\sigma}{r}\right)^{6}\right], \\
& v_{\text {cutoff }}=v \text { for } r=r_{c},
\end{aligned}
$$

where $v$ is the Lennard Jones potential and $r_{c}$ is the cutoff distance which is taken as $2^{1 / 6}$ for surface-surface beads and 2.5 between polymer-polymer beads and between surfacepolymer beads. The interaction parameter $\varepsilon$ is 1 for surfacesurface and polymer-polymer bead interactions and 0.5 for surface-polymer bead interactions.

Bonded interactions are taken to comprise of terms with 2nd and 4th power of the distance between bonded beads:

$$
v_{i j}=\frac{1}{2} k_{1}\left(r_{i j}-r_{e q 1}\right)^{2}+\frac{1}{4} k_{2}\left(r_{i j}-r_{e q 2}\right)^{4},
$$

where, the 2nd power term gives a suitable description of the potential at small deviations from equilibrium bond lengths, while the 4th power term which dominates at larger deviations gives a stiffer restoring force at these distances. The values of $r_{e q 1}, r_{e q 2}, k_{1}$, and $k_{2}$ are 2.47, 2.57, 500, and 60000 for center and surface beads; $0.97,1.07,500$, and 40000 for both surface-polymer and polymer-polymer beads; and 1.00, $1.09,300$, and 40000 for surface-surface beads, respectively. To prevent chains from penetrating into the cores, the core center bead and chain beads interact via a repulsive potential: $v=500(r-2.5)^{2}$ for $r<2.5$, and $v=0$ otherwise.

We used reduced LJ units with $T^{*}=k_{B} T / \varepsilon, P^{*}$ $=P \sigma^{3} / \varepsilon$, and $\rho^{*}=N / V \sigma^{3}$ throughout and employ an inhouse molecular dynamics code which implements the velocity Verlet algorithm where temperature is kept constant at $T^{*}$ $=1.0$ using the Lowe-Andersen thermostat with the coupling constant (fraction of collision pairs whose velocities are adjusted at each time step) set to 0.0003 . The Lowe-Andersen thermostat is used to preserve hydrodynamic interactions. ${ }^{14}$ The equilibration pressure was $P^{*} \approx 0.2$ for systems $\mathrm{B}, \mathrm{C}$, and $\mathrm{D}$, and $P^{*} \approx 0.7$ for systems type A (i.e., A-100, A, and A50 ); this is done to always have a number density for the fluid of $\sim 0.82$ (defined as the ratio of number of chain beads to the volume not occupied by the cores); i.e., a suspending polymer media of equal density as is the case in experiments. All systems were pre-equilibrated by using the Berendsen barostat with a coupling constant as 0.0005 and volume moves enacted after every 15 steps. The time step for simulations is $\Delta t=0.005$.

\section{EVALUATION OF PROPERTIES}

\section{A. Corona thickness and interdigitation}

The corona thickness, $\xi$, is estimated as the distance from the nanoparticle surface at which $90 \%$ of the grafted chain beads are found. This is illustrated in Fig. 4(a) for systems A and $\mathrm{C}$ (see Table I), showing that longer chains have wider, longer-tailed distributions than shorter chains. Interdigitation, 
(a)

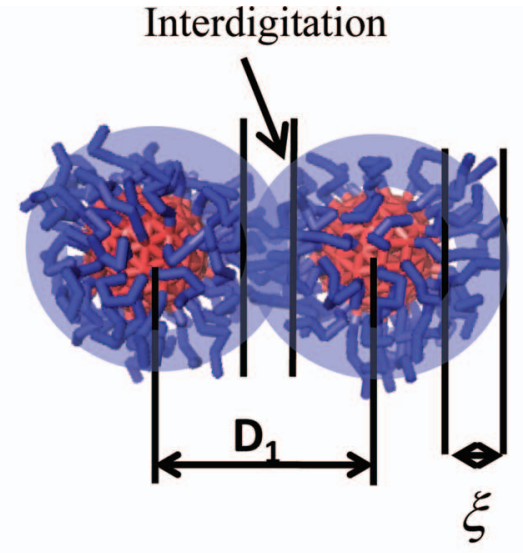

(b)

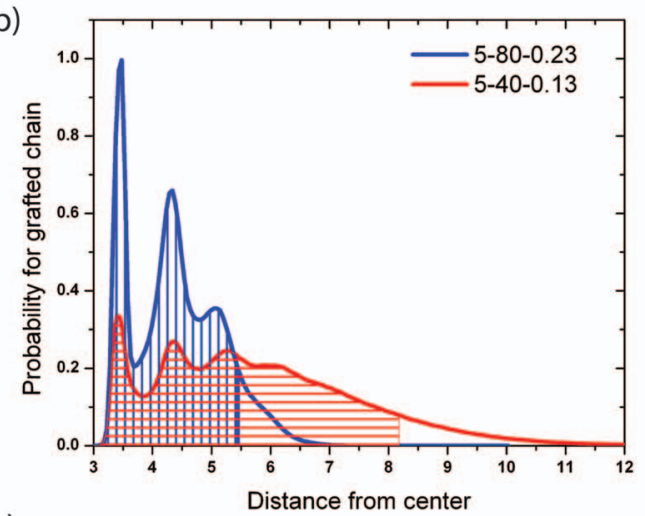

(c)

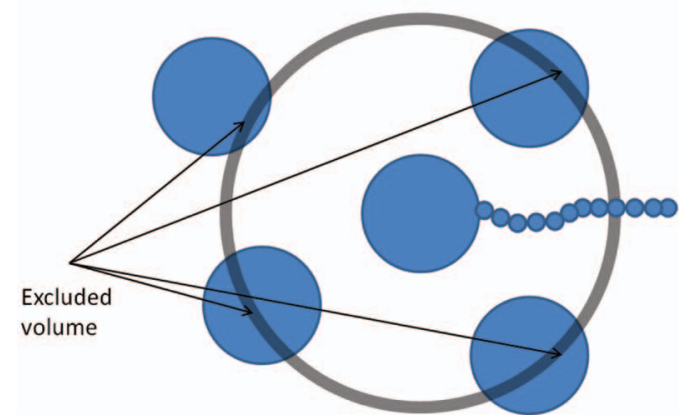

FIG. 4. Schematics of (a) corona thickness and interdigitation in PGNs, (b) probability of finding grafted chain bead from surface of nanoparticles for short and long chains, and (c) volume excluded for calculation of $g_{\alpha c}(r)$ (for $c \neq \alpha)$

a measure of overlap between chains of neighboring particles with respect to corona thickness, is defined as

$$
\text { Interdigitation }=2-\frac{\left(D_{1}-D\right)}{\xi},
$$

where $D_{1}$ is the distance between neighboring cores which is calculated from the location of the first peak of the core-core radial distribution function (Fig. 4(a)); $D_{1}-D$ is the distance between core surfaces.

\section{B. Pair distribution functions}

Three radial distribution functions of interest are the core-core, $g_{c c}(r)$, core-grafted polymers, $g_{c g}(r)$, and core-free polymers, $g_{c f}(r)$. These functions are defined as follows:

$$
g_{\alpha \beta}(r)=\frac{\text { Number of } \beta \text { type particles at distance } r \text { from } \alpha}{\text { Number of ideal gas particles at distance } r \text { from } \alpha} \text {. }
$$

For the calculation of $g_{c g}(r)$ and $g_{c f}(r)$, the denominator contains the number of ideal gas particles in the volume element at distance $r$ that is available for chains to occupy. This volume is obtained by subtracting the volume occupied by nanoparticle cores from each volume element at distance $r$ as shown in Fig. 4(c).

\section{Diffusion}

Translational and rotational diffusivities for various PGN's are calculated by the Stokes-Einstein and StokesEinstein-Debye relations ${ }^{15-17}$ which are given by

$$
\begin{aligned}
D_{t, \text { calculated }} & =\lim _{\Delta t \rightarrow \infty} \frac{1}{6 \Delta t}\left\langle r^{2}(\Delta t)\right\rangle, \\
D_{r, \text { calculated }} & =\lim _{\Delta t \rightarrow \infty} \frac{1}{4 \Delta t}\left\langle\varphi^{2}(\Delta t)\right\rangle .
\end{aligned}
$$

The terms $r^{2}(\Delta t)$ and $\varphi^{2}(\Delta t)$ are the mean squared displacement for position and orientation (angle), respectively. The translational motion is measured by tracking the center of core particles. For the calculation of rotational diffusivity we define a principle unit vector which extends from the center of the nanoparticle to a fixed bead on the surface $\left(\vec{p}_{i}\right)$. The rotation is tracked by the vector angle traced by $\vec{p}_{i}$ in time $\Delta t$, the magnitude of which is given by $\cos ^{-1}\left(\vec{p}_{i, t} \cdot \vec{p}_{i, t+\Delta t}\right)$ and the direction by $\vec{p}_{i, t} \times \vec{p}_{i, t+\Delta t}$. As the cosine function is periodic, we cannot distinguish between rotations of $\theta$ and $(2 \pi+\theta)$ and therefore we calculate $\vec{\varphi}$ by adding the rotation vectors $\delta \vec{\varphi}$ for every time interval between 0 and $t$ to obtain the net angle using

$$
\vec{\varphi}(t)=\int_{0}^{t}(\delta \vec{\varphi}) d t^{\prime}
$$

For a system in diffusive regime, the distribution of particles is given by the Van Hoove distribution and the deviation from this is quantified by using the nongaussianity parameters

$$
\begin{aligned}
& \alpha_{2}(t)=\frac{3\left\langle\varphi^{4}(t)\right\rangle}{5\left\langle\varphi^{2}(t)\right\rangle^{2}}-1, \\
& \alpha_{2}(t)=\frac{3\left\langle r^{4}(t)\right\rangle}{5\left\langle r^{2}(t)\right\rangle^{2}}-1,
\end{aligned}
$$

for rotation and translation, respectively. Nongaussianity parameter increases from ballistic to sub- diffusive regime and attains maxima when system enters diffusive regime. We used the location of the peak of this function to identify crossover to diffusion for our systems. ${ }^{18}$

The absolute diffusivity values reported are in reduced LJ units. To facilitate comparison to experiments, a ratio of the calculated diffusivity is taken with respect to the ideal diffusivity of an identical nanoparticle (core) in a polymer melt as calculated by the Stoke-Einstein and Stoke-Einstein-Debye relations given by

$$
D_{t, \text { ideal }}=\frac{k_{B} T}{6 \pi \eta R} ; D_{r, \text { ideal }}=\frac{k_{B} T}{8 \pi \eta R^{3}},
$$


where $T$ is the temperature, $\eta$ is the zero shear viscosity of the fluid, $k_{B}$ is the Boltzmann constant, $R$ is the radius of nanoparticle, and the polymer melt is taken to consist of chains of the same length as those grafted to the PGNs. We refer to this ratio of diffusivity to ideal diffusivity as the relative diffusivity:

$$
D_{t}=\frac{D_{t, \text { calc }}}{D_{t, \text { ideal }}} ; D_{r}=\frac{D_{r, \text { calc }}}{D_{r, \text { ideal }}} .
$$

\section{Constant-rate uniaxial extension}

Simulations that probe the dynamic response of the system to a uniaxial deformation have often been used to characterize the strength and failure of adhesives and glassy polymers. ${ }^{19-21}$ We implemented this technique in our system having periodic boundary conditions (along all axes) by gradually elongating the box along the $z$ axis while keeping the $x y$ cross section constant. The system starts inside a cubic box and is pre-equilibrated at $T^{*}=1.0$ and a density consistent with $P^{*}=0.2$. While the dynamic response depends on the strain-rate applied, we adopt here a single rate value of $0.02 \sigma / \Delta t$ (perturbations enacted every 100 equilibration steps) which is consistent with typical values used with such simulations for polymeric materials. ${ }^{21}$ As the box elongates, "volume" is being created which forces the system to eventually become heterogeneous and break up into filled and empty domains. This constant cross-section uniaxial stretching differs from constant-volume stretching experiments (where the sample cross-section is concertedly reduced); the former is indicated to probe the failure of glassy films with relatively large $x y$ cross-section, while the latter is more suited to probe the elastic response of rubbery materials.

\section{E. Viscosities and yield stress}

For calculating the shear-dependent viscosity of the systems, SLLOD, ${ }^{22,23}$ a commonly used non-equilibrium molecular dynamics (NEMD) technique is used along with Lees-Edwards boundary condition. ${ }^{24}$ SLLOD imposes a homogenous steady shear strain on the system and measures the resulting steady state stress. Similar to diffusivities, we reduce the viscosities by a factor $\eta_{0}$ which is the zero-shear viscosity of the melt comprising of chains identical to the grafted polymers, to obtain relative viscosities $\eta_{\text {rel }}=\eta_{\text {actual }} / \eta_{0}$. To relate our results with experiments, we convert shear rates to a ratio of convective motion to diffusive motion given by the Peclet number $(\mathrm{Pe})$ defined as

$$
\mathrm{Pe}=\frac{6 \pi \eta_{0} \gamma R^{3}}{k_{B} T},
$$

where $\gamma$ is the shear rate and $R$ is the core radius. To estimate yield stresses we use the constant stress algorithm of Hood, Evans, and Morriss ${ }^{25}$ (using their Eq. (3) and adopting a value of $\tau_{y} .=100$ for the feedback response time).

We study the effect of corona thickness and GD on viscosity and yield stress. We vary the corona thickness by changing chain length and GD for pure PGNs. Chain length is varied while keeping $\phi_{c}$ constant to help us isolate and understand the effect of corona thickness on both equilibrium and rheological properties.

\section{F. Brownian stress}

On application of shear, the flow induced disturbance in the system perturbs the equilibrium structure; the resulting particle diffusion induces a stress. ${ }^{26}$ The viscous contribution of this stress is known to reduce with the Peclet number thus causing shear thinning. This so-called Brownian stress is calculated by ${ }^{26-28}$

$$
n\left\langle S^{B}\right\rangle=-n^{2} k_{B} T R \int_{r=2 a} \hat{r} \hat{r} g(\vec{r}) d \Omega
$$

where $n$ is the number density of nanoparticles in the system, $R$ is the particle radius taken as the radius of nanoparticle $(\approx 3$ $\sigma), g(\vec{r})$ is the radial distribution function at $\vec{r}$, and $\hat{r}$ is the unit vector of $\vec{r} \cdot g(\vec{r})$ is calculated by storing the separation vectors between pairs of particles and creating a histogram of these vectors on a grid of spherical coordinates $r, \theta$, and $\phi$ and normalizing it by the number of ideal gas particles in the same volume element:

$g(r, \theta, \phi)$

$=\frac{\text { Number of PGN centers in elemental volume at } r, \theta, \phi}{\text { Number of ideal gas particles in elemental volume } V(r, \theta, \phi)}$,

where $V(r, \theta, \phi)=r^{2} \sin \theta d r d \theta d \phi$, we have taken only the contact integral into account as it is known to give an accurate description of the Brownian stress. ${ }^{26,27}$ Equation (12) was developed for colloidal particles suspended in a continuum fluid and hence it is not strictly applicable to pure PGNs; however, it is expected to give a semiquantitative description of Brownian stresses for PGNs given that the grafted chains provide a relatively homogeneous (self-suspending) media for the cores.

\section{G. Chain orientation}

Due to shear, grafted and free chains (if present) in PGN systems tend to align in the direction of shear; this makes the polymers more streamlined and reduces viscosity. ${ }^{29,30}$ To study this phenomenon in our systems, we calculate the angle $\left(\theta^{\prime}\right)$ made by the end-to-end vector of each polymer with the shear axis (the $x$ axis here, see Fig. 5). A probability distribution for $\theta^{\prime}$ is obtained from

$$
f_{\theta^{\prime}}=\frac{P_{\theta^{\prime}}}{\sin \theta^{\prime}}, \text { where } P_{\theta^{\prime}}=\frac{\text { Frequency of angle } \theta^{\prime}}{\text { Total number of polymers }} .
$$

$P_{\theta^{\prime}}$ has been divided by $\sin \theta^{\prime}$ to obtain the ratio with respect to the ideal distribution which is proportional to the solid angle being sample around $\theta^{\prime}$; i.e., $\sim \sin \theta^{\prime} d \theta^{\prime} d \phi$. Note that Eq. (14) will produce a flat distribution for an isotropic distribution of angles but will tend to overemphasize $P_{\theta^{\prime}}$ for small values of $\sin \theta^{\prime}$. 


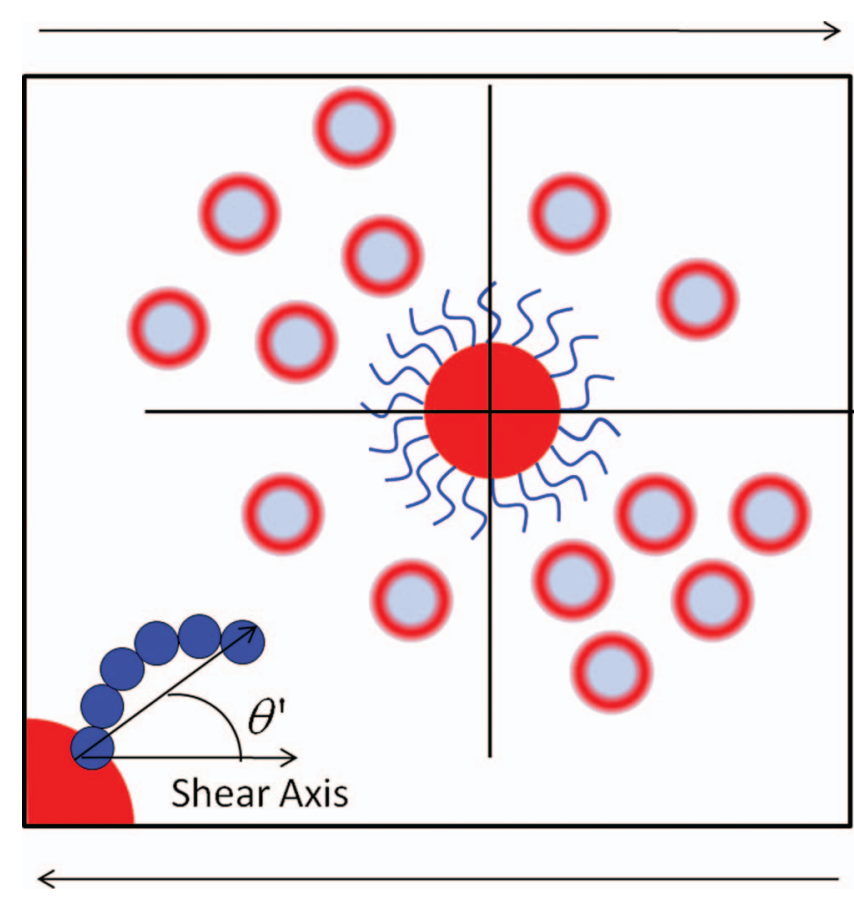

FIG. 5. Schematic of anisotropic distribution of particles under shear and definition of chain orientation angle under shear $\theta^{\prime}$.

\section{RESULTS AND DISCUSSION}

\section{A. Structure and diffusivity}

To understand the effect of core concentration or $\phi_{c}$, we calculate the relative diffusivity of a single PGN with $\mathrm{N}_{\mathrm{m}}$ $=5$ and $\mathrm{GD}=80 \%$ in a melt of $\mathrm{N}_{\mathrm{m}}=5$, i.e., in our notation it is given by 5-80-0(5-1). The relative diffusivity of this single PGN isolates the effect of tethering chains on the nanoparticle and is not affected by caging or interdigitation. The value of the relative translational diffusivity is 0.55 for such a single PGN which is much larger than the value of 0.0052 obtained for a system of pure identical PGNs (system A: 5-80-0.19). This shows that with the addition of more cores, the particles get caged and translational diffusivity is reduced. For rotation, the relative diffusivity for a single PGN in the chain melt is 0.36 which is close to the diffusivity of 0.16 for system $\mathrm{A}$, indicating that the friction between tethered chains is only slightly larger than that between tethered and free chains.

We focus next on the effect of corona size for a fixed value of core volume fraction. The systems under study are system B (10-80-0.13), C (20-40-0.13), and D [5-80-0.13(5$0.5)$ ], where always $\phi_{c}=0.13$ while tethered chain length varies. Figure 6(a) shows the core-core radial distribution functions for these systems. System C shows a very even $g_{c c}(r)$ with a short first peak, consistent with a well dispersed liquid-like system, while system B shows a more pronounced structure (higher peaks and deeper troughs) suggestive of a more ordered material. System D [5-80-0.13(5-0.5)] constitutes an intermediate case as the free chains can here spread out more evenly than the attached chains in system B. This is also supported by Fig. 6(b) which shows the distribution of $g_{c c}(r), g_{c g}(r)$, and $g_{c f}(r)$ for system D, where the latter distri-
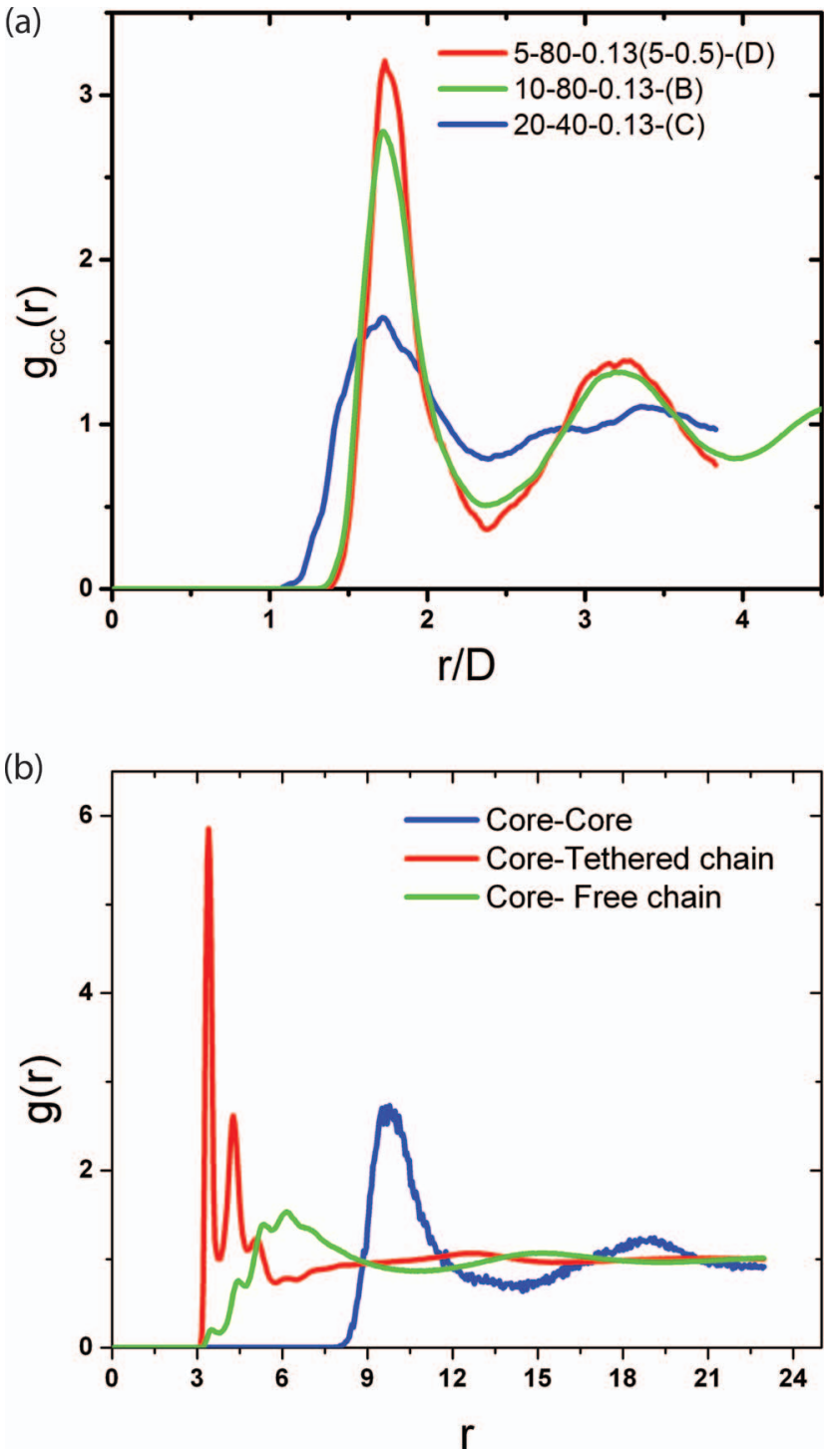

FIG. 6. (a) Radial distribution function for system B, C, and D where $\phi_{c}$ $=0.13$ is fixed while chain length varies. (b) Distribution functions for cores, tethered, and free chains in the blend system D.

bution of free chains is observed to be close to that of the ideal gas.

Table II lists the values of diffusivities and interdigitation for systems A, B, C, and D. For system C the interdigitation is greater than 1 , indicating that chains extend beyond the nearest neighboring cores due to the long tail of the corona thickness distribution $(\xi)$. From the relative diffusivity we can clearly see that for pure PGNs, translational motion increases significantly as chain length increases $(\mathrm{A}<\mathrm{B}$ $<$ C) while rotational motion remains almost unchanged. This supports our earlier observation that when the chains become longer, the corona becomes more uniformly dispersed, caging of the nanoparticles is reduced, and translational diffusivity increases. The enhanced mixing of chains from neighboring particles with longer chains is also evident in the increasing values of interdigitation for systems $\mathrm{B}$ and $\mathrm{C}$. The rotational diffusivity remains almost constant in all cases and is unaffected by any change in corona, possibly because interdigitation values remain nearly constant resulting in a similar 
TABLE II. Effect of variation in corona on diffusivities for various systems.

\begin{tabular}{|c|c|c|c|c|c|}
\hline System /Diffusivities & Translation (Absolute) & Translation (Relative) & Rotation (Absolute) & Rotation (Relative) & Interdigitation \\
\hline A & $2.36 \times 10^{-5}$ & 0.0052 & $2.0 \times 10^{-5}$ & 0.16 & 0.95 \\
\hline B & $1.47 \times 10^{-4}$ & 0.066 & $1.95 \times 10^{-5}$ & 0.078 & 0.86 \\
\hline $\mathrm{D}$ & $4.42 \times 10^{-4}$ & 0.0812 & $1.375 \times 10^{-5}$ & 0.11 & 0.50 \\
\hline $\mathrm{C}$ & $9.7 \times 10^{-4}$ & 0.76 & $2.39 \times 10^{-5}$ & 0.17 & 1.17 \\
\hline A-50 & $5.35 \times 10^{-5}$ & 0.012 & $3.5 \times 10^{-5}$ & 0.24 & Some cores touch \\
\hline A-100 & $1.18 \times 10^{-5}$ & 0.0026 & $1.40 \times 10^{-5}$ & 0.11 & 0.76 \\
\hline
\end{tabular}

effective "friction" of a PGN with its surroundings. In Fig. 3(c), systems B and C are simply marked as liquids while system A is loosely marked as a "soft glass" due to the strong caging of its cores. Both translation and rotation diffusivities of the blend system D are slightly larger than those for system $\mathrm{B}$ but less than those of system C; this result and those for the equilibrium structure discussed before suggests that blends exhibit a more uniformly dispersed corona compared with a pure PGN system of the same grafting density and $\phi_{c}$. This can be attributed to the extra mobility of free chains which allows the blend (D) to relax more readily.

We also studied the effect of changing GD with fixed $\mathrm{N}_{\mathrm{m}}$ on the structure and mobility of pure PGNs. We used systems A-50, A, and A-100 where we have kept $\mathrm{N}_{\mathrm{m}}=5$ and changed GD from 50 to 100 (see Fig. 3(c) and Table I). Table II and Fig. 7 give the simulated properties. We see in Fig. 7(a) that compared to system A, the thicker corona in the A-100 nanoparticles leads to a larger distance between neighbors and a taller first peak of $g_{c c}(r)$. Effectively, the A100 system experiences stronger steric hindrance due to extra grafted chains making the net particle-particle interaction more repulsive and the lubricating corona less fluid-like. This causes resistance for interparticle motion and a reduction in translational and rotational diffusivity. For the system A-50, a thinner corona is observed along with a number of core pairs "kissing" at a distance of $6 \sigma$ which is the diameter of the core. This arises as regions with fewer tethered chains or "bald spots" of two PGN surfaces come together experiencing a depletion-induced attraction (associated with the grafted chains). It should be noted that the grafting of chains at random points on the core surface is bound to produce some patchiness in the corona for lower GD; hence, the results for system A-50 will depend on the specific choices of chain anchoring points (though the effect is mild for relatively uniform spreading). Despite having a large $\phi_{c}(=0.32)$, the diffusivities for system A-50 are larger than those for systems A and A-100. This is explained by the decrease in the structural layering of the cores as $\phi_{c}$ increases; the presence of "kissing cores" in system A-50 seems to further contribute to the disordering of cores and to a more uniform distribution of chains (both of which reduce the caging of particles).

\section{B. Viscosity and yield stress}

To examine the effect of GD on relative viscosities, we simulated the systems A-50 (5-50-0.32), A (5-80-0.23), and A-100 (5-100-0.19) under shear (see Fig. 7(b)). As noted earlier, the end-to-end distances of grafted chains increase with
GD, seemingly increasing the effective friction between particles. This results in an increase of relative viscosities for system A-100 relative to system A (80\% GD). For system A-50 (5-50-0.32) we observe the lowest viscosities for PE $<6$, likely because at low shear rates some particles stay aggregated (as in the equilibrium state) reducing the steric hindrance for the other particles and facilitating the overall flow.

(a)
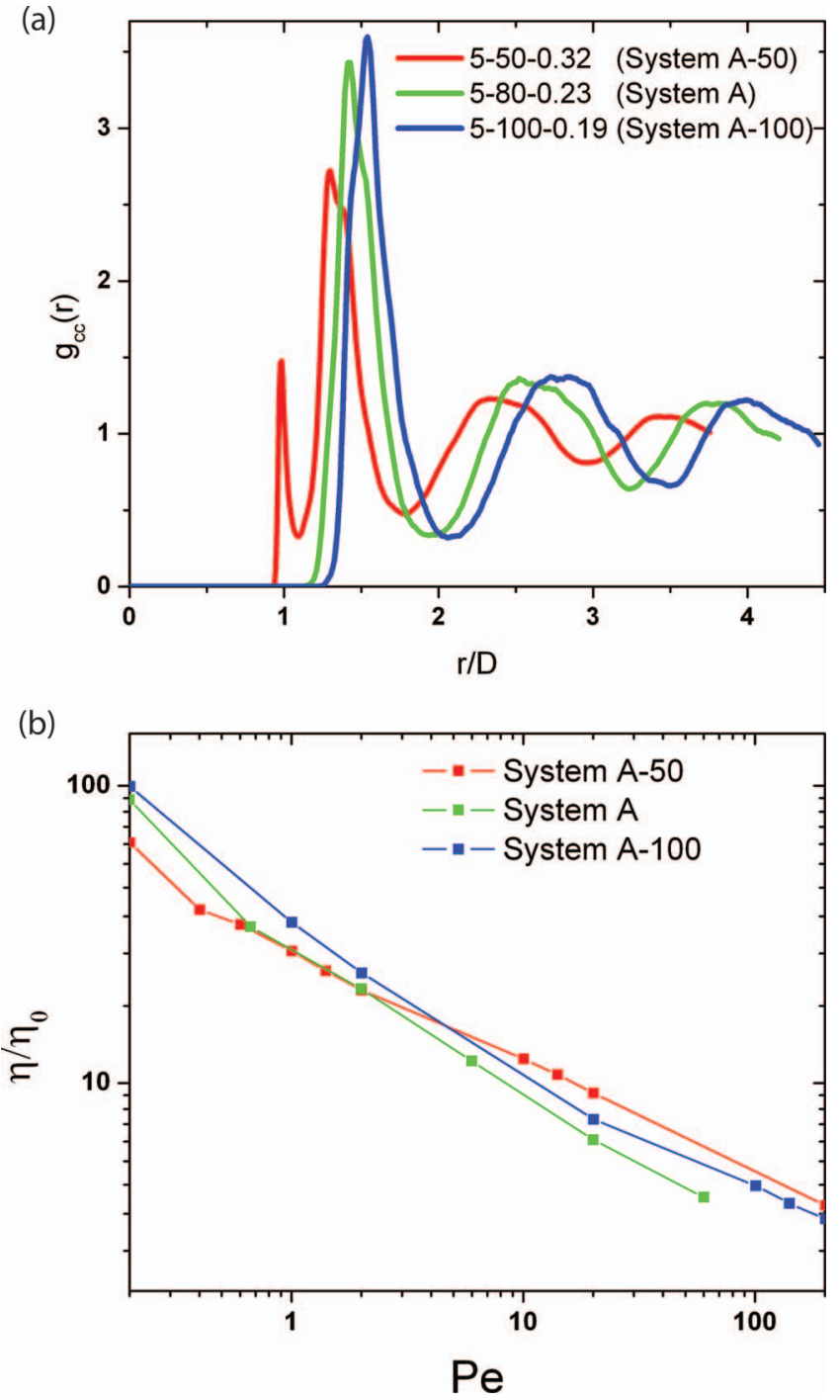

FIG. 7. Properties of systems A, A-50, and A-100 (which only differ in GD and hence $\phi_{c}$ ). (a) Core-core radial distribution function; particles aggregate at lower GD due to bald core surfaces sticking together. (b) Viscosities as a function of Peclet number where a transition behavior occurs for system A-50 as large Pe induces the separation of "kissing" particles. 

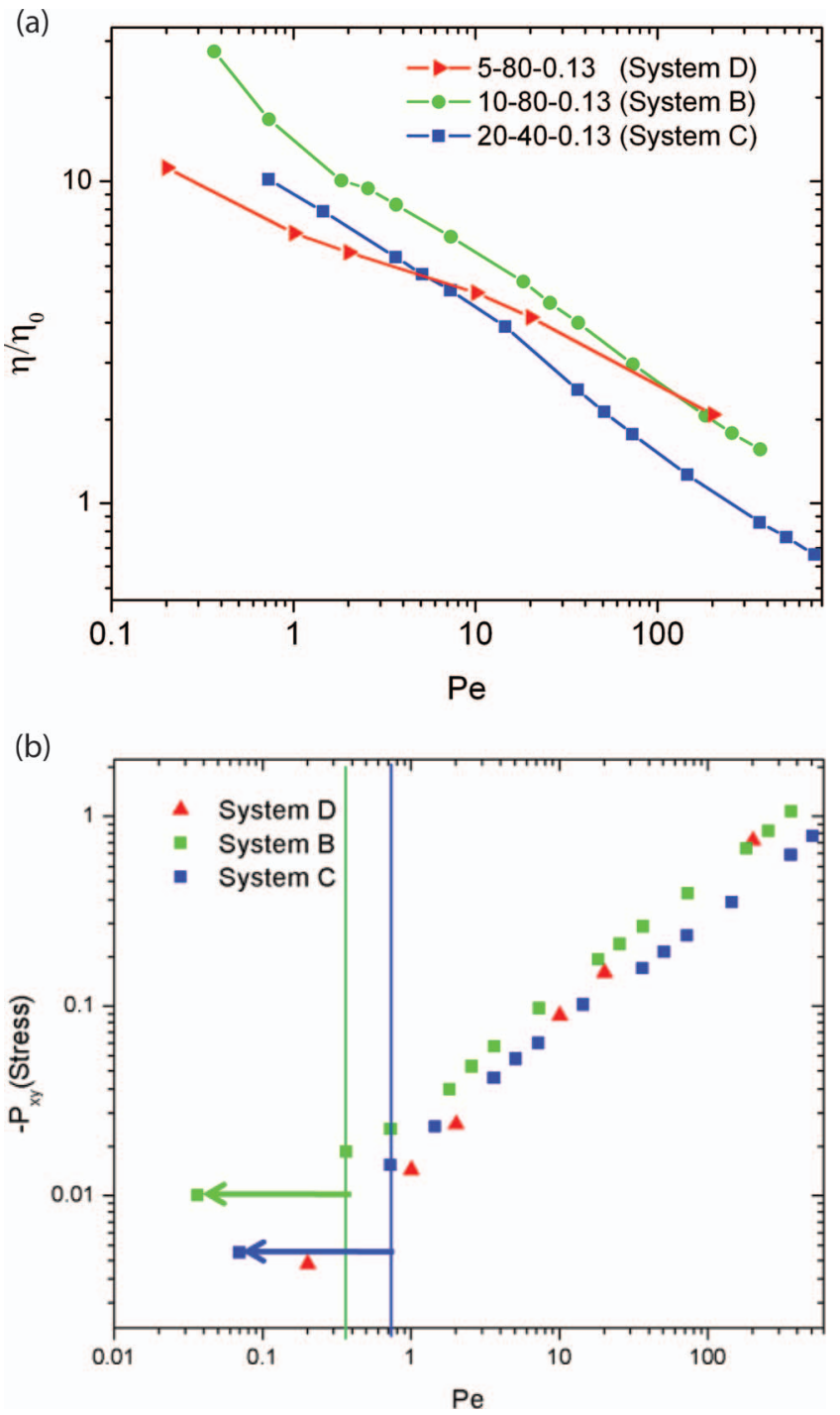

FIG. 8. (a) Viscosity for systems D, B, and C as a function of Peclet number via constant shear-rate flow type simulation. (b) Yield stresses for systems D, $\mathrm{B}$, and $\mathrm{C}$ via constant stress type simulations.

As $P e$ increases, the shear applied is enough to separate the particle aggregates and the viscosity becomes larger than that of the systems with lower $\phi_{c}$.

System A-50 is marked as "aggregating fluid" in Fig. 3(c) as the tendency to form core-core contacts is the most distinctive characteristic. While all systems A are liquids based on their translational diffusivity behavior, systems A and A100 are tentatively termed "soft glasses" in Fig. 3(c) to highlight their marked caged structure, relatively low translational diffusivity, and, a large low-Pe viscosity (further evidence for system A-100 is given in Sec. IV D). This classification is in approximate agreement with the trends observed in Ref. 12.

To study the effect of corona variation at fixed $\phi_{\mathrm{c}}=0.13$ on viscous response, we simulated the viscosities for systems B, C, and D as shown in Fig. 8. Shear thinning behavior is observed for all cases. This behavior is consistent with experiments though we have not approached the range of Pe where Newtonian regime is observed for experiments. ${ }^{2}$

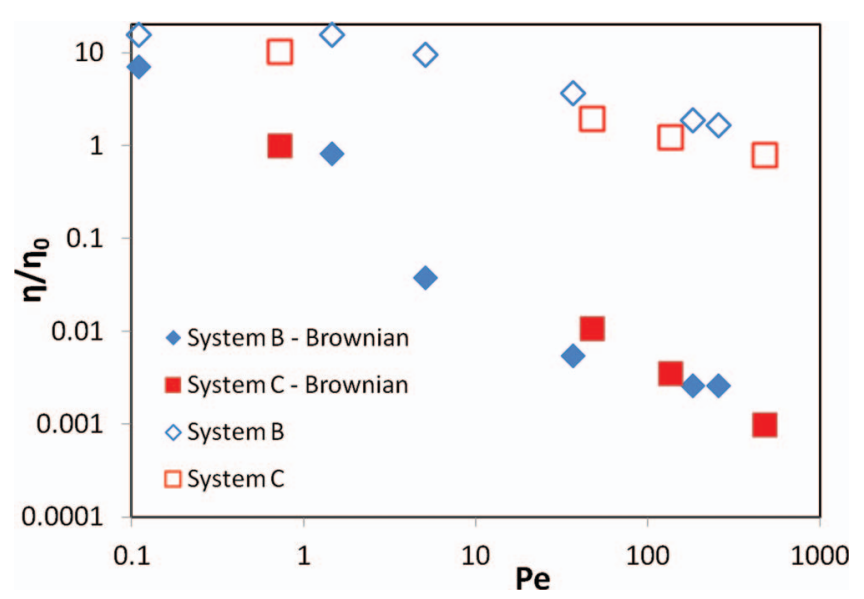

FIG. 9. Comparison of actual viscosity and viscous contribution of Brownian stresses for systems B and C. Both systems shear thin but the relative contribution of Brownian stress is negligible at higher Pe.

With increasing chain length at fixed $\phi_{c}=0.13$, we observe a decrease in relative viscosity with system $C$ always having lower viscosity than system B. Both pure systems follow power law behavior with an exponent of -0.4 , while the blend system D has a lower exponent of -0.25 . At high Pe all systems tend to have more similar relative viscosities due to chains being strongly aligned to the direction of flow (see Sec. IV C). As this simulation method is not efficient to obtain low-Pe results, we also calculated the yield stress behavior. Figure 8 shows the stress versus shear rate curves for these systems along with the yield stress and yield strain. We observe that the pure systems require a finite stress to yield while the blend does not show a clear yielding behavior. The region marked by the arrow shows the regime that was not accessed by our constant shear-rate simulations.

\section{Shear thinning}

\section{Brownian stresses}

Figure 9 shows the relative viscous contribution due to Brownian stresses and relative viscosity as a function of the Peclet number. The Brownian stresses show a uniform shear thinning behavior similar to that of the total viscosities and can be considered as one of the contributors to shear thinning. Such an effect, however, is very small as evident from the ratio of Brownian contribution to total viscosity which is $\sim 10^{-3}$ for the $1<\mathrm{Pe}<1000$. This occurs because Peclet numbers accessible to simulation are very large which by definition means a large ratio of convective to Brownian forces; hence Brownian forces are weak and have negligible effect on dynamics for Pe $\gg 1$.

\section{Chain alignment}

We next examine results of the chain orientation function $f_{\theta^{\prime}}$ (Fig. 10). Figure 10(a) plots $f_{\theta^{\prime}}$ for increasing Pe: for $\mathrm{Pe}=0$ (at equilibrium) there is no preferential chain alignment, for $\mathrm{Pe}=0.25$ some alignment is evident, while for Pe $=50$ chain alignment is very significant. Figure 10(b) shows 

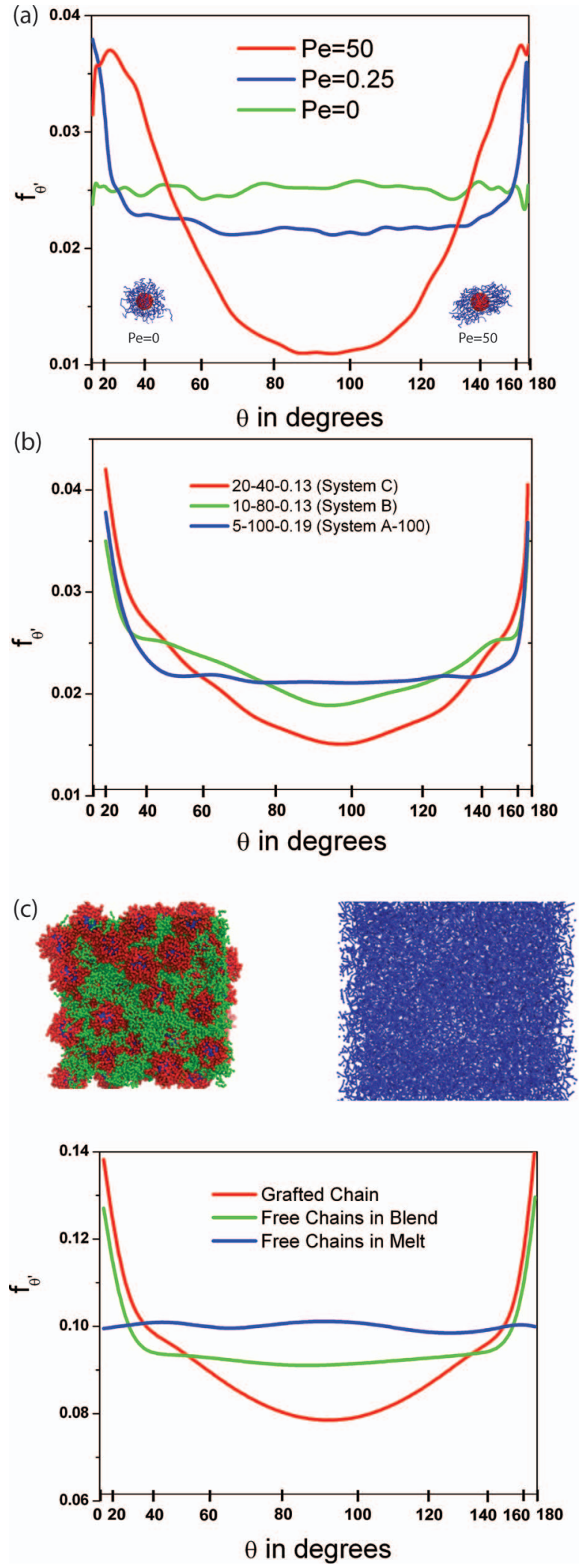

FIG. 10. Chain orientation function $f_{\theta^{\prime}}$ for various cases. Note the non-linear scale used for the $x$ axis to maintain a proper area normalization of $f_{\theta^{\prime}}$. (a) Variation of $f_{\theta^{\prime}}$ with Pe number for system B showing distortion of corona at high Pe. (b) Variation of $f_{\theta^{\prime}}$ with chain length for a fixed shear rate $(\gamma$ $\Delta t=3.5 \times 10^{-6}$ ) showing more alignment for longer chains. (c) Variation of $f_{\theta^{\prime}}$ for different types of chains in the blend system $\mathrm{D}$ and in a pure 5 -mer melt (fixed $\mathrm{Pe}=10$ ) showing that grafted chains (red curve) align the most. Snapshots show the two systems with colors corresponding to $f_{\theta^{\prime}}$ curves. $f_{\theta^{\prime}}$ for systems $\mathrm{A}, \mathrm{B}$, and $\mathrm{C}$ at a fixed shear rate. We observe that longer chains align more easily than shorter chains. This partly explains why system $C$ has lower viscosities than systems B and A. Figure 10(c) plots $f_{\theta^{\prime}}$ for both grafted chains and free polymers in the blend system D at a fixed Pe of 10 . We see that grafted chains align more than the free chains, which is likely due to the former having less freedom to reorient isotropically. Also shown in Fig. 10(c), the free chains in the blend exhibit an extent of alignment which is intermediate between that of the grafted chain and that of the polymers in a melt (free of PGNs). Overall, these results indicate that chain alignment is one of the causes for shear thinning, especially at high Pe numbers, and blends have a lower shear thinning slope because the free chains orient less in the direction of shear than grafted chains.

To elucidate the coupling between the spatial distribution of cores and the alignment of chains, we examined the existence of a "residual" chain alignment as described next. When the system is under shear, the equilibrium core-core distribution is altered to a non-equilibrium state (Fig. 11(a)) and polymers may have to orient anisotropically maintaining some of the shear-induced alignment to fill the space between nanoparticles (thus maximizing the chain conformational entropy). To quantify this residual chain alignment, we carry out the following steps: (1) starting with an equilibrium system, steady shear is applied until steady state is reached, (2) the position of all the core centers is frozen and the shear is turned off to only allow the chains to relax (cores are also allowed to rotate). Figure 11(b) shows the residual alignment of chains after shear has been turned off for system B. Figure 11(c) plots the residual alignment for various Pe numbers and we observe that it increases with Pe. We can then conjecture that shear thinning at these Pe numbers is due to a coupled effect of chain alignment arising from both the shear flow and a residual alignment associated with the non-equilibrium spatial distribution of cores.

\section{Uniaxial stretching and breakup}

We simulated the pure PGN systems A-100 and B-50 which possess the same core volume fraction $\left(\phi_{c}=19 \%\right)$ but differ in grafted chain length. Representative results are shown in Fig. 12. As the box elongates and its volume increases, a large negative force (tension) along the $z$ axis is generated (to sustain the deformation) while the system's density drops but remains uniform; this large tension eventually leads to defects that seed a very low density region (vacuum). The tension then starts to drop as the empty region grows and eventually vanishes as the dense (liquid) domain becomes fully separated by a "gas-like" interface. While the maximum in the tension vs. strain curve is similar (in height and location) for both systems, the post yield-point behavior is significantly different: the B-50 system (having the longer 10-mer grafted chains) forms a characteristic liquid bridge before a breakup that takes place at a much longer extension. In contrast, the A-100 system (with the shorter 5-mer chains) breaks up earlier and more transversally with a minimal bridge; i.e., a behavior more reminiscent of that of a soft glass or solid. This 

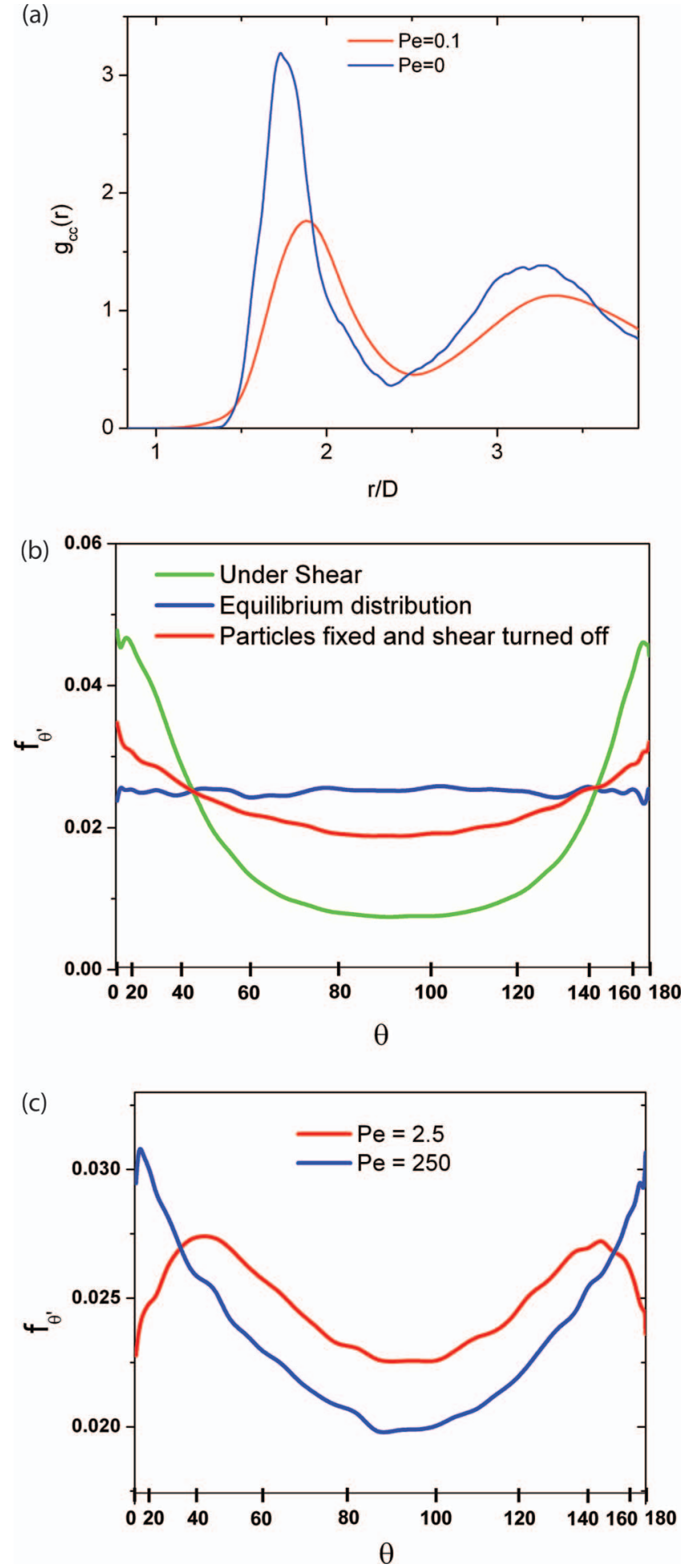

FIG. 11. Effect of shear on system B. (a) Radial distribution function under equilibrium and at $\mathrm{Pe}=0.1$ showing the perturbation in structure leading to residual chain alignment. (b) Residual chain alignment when system is equilibrated with cores held fixed at the $\mathrm{Pe}=0.1$ structure. (c) Increase in residual chain alignment with $\mathrm{Pe}$.

liquid vs. glassy-like behavior agrees well with the difference in the core structure (shown in the inset of Fig. 12) where the B-50 has a much less structured and layered radial distribution than system A-100. These results are in line with the analysis of systems B and C (for a lower $\phi_{c}=0.13$ ), the correlations

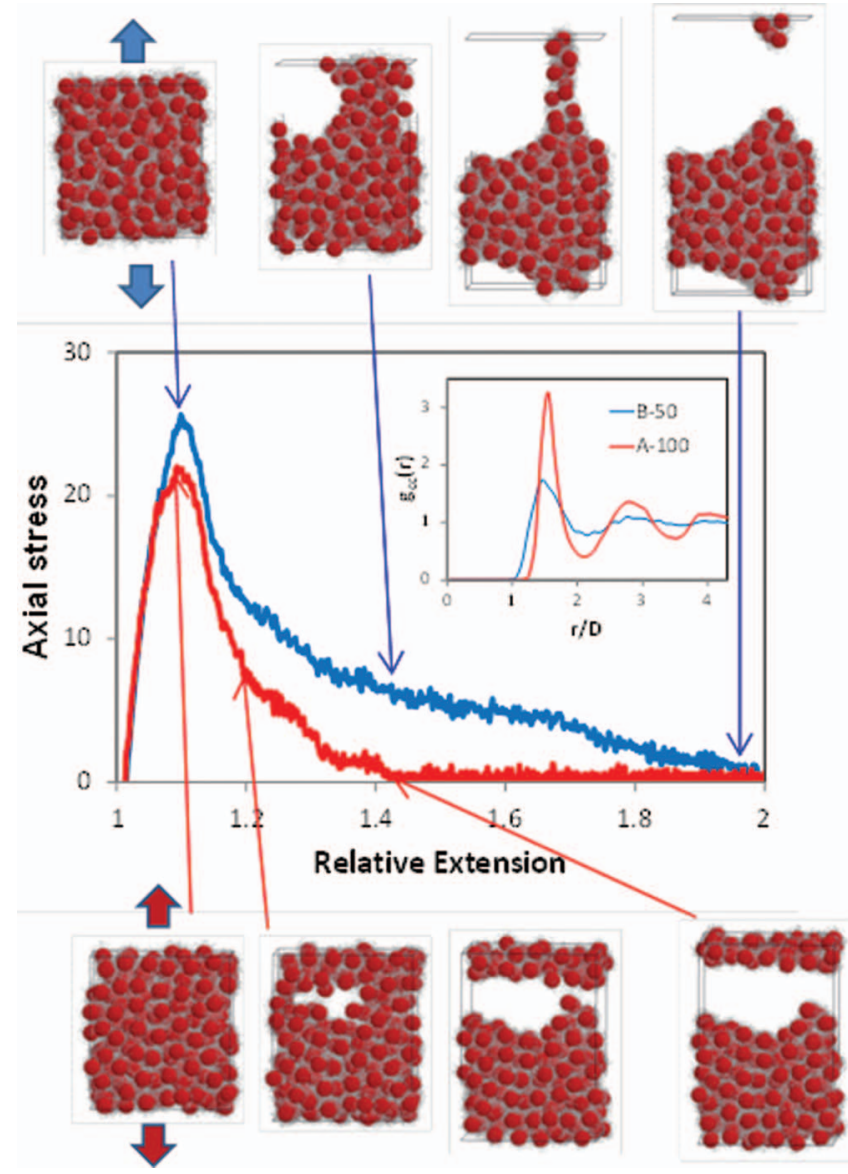

FIG. 12. Constant cross-section uniaxial deformation of systems A-100 and B-50. The axial stress is the product of the instantaneous $z z$ component of the stress tensor and the box $z$-length (in LJ units); the relative extension is with respect to the initial unperturbed box length.

between their diffusivities and structure described in Sec. IV $\mathrm{A}$, and their rheological behavior described in Sec. IV B.

\section{CONCLUDING REMARKS}

In this work, we have studied the equilibrium behavior, diffusivities, and rheology of PGNs as self-suspended colloidal systems for varying GD and $\mathrm{N}_{\mathrm{m}}$, while keeping fixed the core diameter. Comparing a system of pure PGNs with the one in which a single PGN is infinitely diluted in an oligomer identical to the grafted chains, we found that the translational motion is greatly reduced due to the caging by neighboring PGNs, while rotational motion is only mildly reduced due to the lower mobility of the grafted oligomers. Depending on the combination of GD and $\mathrm{N}_{\mathrm{m}}$ employed, pure PGNs exhibited distinctive fluid behavior that is qualitatively highlighted by different symbols in Fig. 3(c). For combinations of GD and $\mathrm{N}_{\mathrm{m}}$ that result in a fixed $\phi_{c}$, we found that for shorter grafted chains the spatial distribution of cores becomes more structured (with better defined neighboring layers around a central core), the translational motion is reduced due to stronger caging by neighboring PGNs, and the rotational motion is almost unaffected. Consistently, simulations of uniaxial stretching (also for fixed $\phi_{c}$ ) show that systems with a longer chain length present a liquid-like breakup while the short chain 
system exhibits a soft-glass-like breakup. For fixed grafted chain length $\mathrm{N}_{\mathrm{m}}$, a reduction of GD (which also brings a corresponding increase in $\phi_{c}$ ) leads to a non-trivial trend in core structure where for low enough GD some particle cores get in close contact (touching in areas of lowest GD) promoting faster translational and rotational diffusivities. Such close contacts also lead to low-GD systems to be less viscous than high-GD systems, a trend that is reversed as the shear rate is increased and the structure made more homogeneous.

Our constant-shear rate simulations revealed that all systems studied exhibit shear thinning behavior with the thinning exponent being greater for pure PGNs than for a blend system of equal $\phi_{c}$. We found that systems exhibiting a more homogeneously dispersed corona have lower viscosities. Also, the introduction of free oligomer fluid to PGNs reduces the viscosity relative to pure PGNs of equivalent $\phi_{c}$, at least in the range of shear rates we have simulated. The yield stress results are consistent with the stress-strain rate trends of the constantshear rate simulations and also show that the blend system did not exhibit a clear yielding behavior. We also attempted to elucidate the microscopic origins of the observed shear-thinning behavior. We found that although Brownian stresses decrease with shear rate, their viscous contribution is too small at the high Peclet numbers simulated to be a dominant driving force of shear thinning. When examining the extent of alignment of chains under shear, we find that systems with more uniformly dispersed corona tend to align more in the direction of shear, giving a possible mechanism for the lower viscosity of PGNs with longer chains. We also found chain alignment to be more pronounced for grafted polymers than for free polymers which partly explain the flatter shear thinning slope of the PGN-polymer blend compared to pure PGN systems. Finally, we observed a residual chain alignment present in PGNs due to the shear-induced non-equilibrium distribution of cores, which must also affect the viscous response of the system.

An important question that arises is to what extent the properties of the PGN systems studied here could be described by using much coarser force fields; e.g., using a softsphere pair potential to represent the effective core-core potential of mean force. Indeed, our results that softening of the PGN corona (e.g., by grafting longer chains) leads to weakened core-core pair correlations and an increase in translational diffusivity have also been observed in simulations of Gaussian-core and Hertzian fluids. ${ }^{31-33}$ Our more detailed PGN model, however, allows us to account for other effects that are associated with multibody intra- and inter-chain interactions, and with intrinsic or dynamic spatial anisotropies of the corona. For example, our model allowed us to probe rotational diffusivities, to find a connection between shear thinning, chain alignment, and corona deformation, and to find depletion-driven close contacts between bald-spots of cores having low GD. Such effects could also be captured by a coarser-grained model than ours, e.g., one using fewer effective grafted chains that have fewer but larger beads representing longer chain blobs (with softer interactions); more research would be needed to define such a minimalistic model.

This study is a first step toward understanding the physical behavior of the solvent-free PGNs of varying designs. In terms of thermodynamic behavior, it is still unclear, for example, whether and how a liquid-to-solid transition ensues. In terms of rheological behavior, we were only able to probe Pe values as low as 0.1 , while interesting experimental crossover behavior (to a Newtonian regime) of related systems is usually reached at much lower Pe values $(\sim 0.0001) .{ }^{3}$ While constant shear-rate simulations for $\mathrm{Pe} \sim 0.001$ could be attainable via very long simulations in massively parallel computing platforms, these may still be in the shear-thinning regime. Regarding our high-Pe shear thinning results, we note that the viscous contribution of chain alignment to stress could be further explored by calculating the pulling force applied by the chains on the nanoparticles. Also, our study has been confined to very small core sizes and monodispersed systems; polydispersity in particle size, chain length, and grafting density is non-negligible in experimental systems and can significantly affect the structural and rheological behavior. Lastly, nanoparticles of non-spherical shapes will likely result in a vastly different viscous behavior. Work on some of these extensions is currently under way

\section{ACKNOWLEDGMENTS}

This paper is based on work supported in part by Award No. KUS-C1-018-02, made by King Abdullah University of Science and Technology (KAUST). It was also supported by Award No. CBET-1033349 from National Science Foundation (NSF). The authors are grateful to Professor D. L. Koch, Professor L. A. Archer, Professor I. Cohen, Professor A. Z. Panagiotopoulos, Dr. Xiang Chen, U. Agarwal, S. Srivastava, and P. Agarwal for useful discussions and suggestions.

${ }^{1}$ R. Krishnamoorti and R. A. Vaia, J. Polymer Sci., Part B: Polymer Phys. 45, 3252 (2007).

${ }^{2}$ A. B. Bourlinos, S. R. Chowdhury, R. Herrera, N. Chalkias, D. D. Jiang, L. A. Archer, and E. P. Giannelis, Adv. Funct. Mater. 15, 1285 (2005).

${ }^{3}$ P. Agarwal, H. Qi, and L. A. Archer, Nano Lett. 10, 111 (2010).

${ }^{4}$ R. Rodriguez, R. Herrera, L. A. Archer, and E. P. Giannelis, Adv. Mater. 20, 4353 (2008).

${ }^{5}$ H.-Y. Yu and D. Koch, Langmuir 26, 16801 (2010).

${ }^{6}$ L. A. Archer and D. Kim, Langmuir 27, 3083 (2011).

${ }^{7}$ D. L. Green and J. Mewis, Langmuir 22, 9546 (2006).

${ }^{8}$ A. Jayaraman and K. S. Schweizer, Macromolecules 42, 8423 (2009).

${ }^{9}$ A. Jayaraman and K. S. Schweizer, J. Chem. Phys. 128, 164904 (2008).

${ }^{10}$ M. E. Mackay, A. Tuteja, P. M. Duxbury, C. J. Hawker, B. V. Horn, Z. Guan, G. Chen, and R. S. Krishnan, Science 311, 1740 (2006).

${ }^{11}$ P. Akcora, H. Liu, S. K. Kumar, J. Moll, Y. Li, B. C. Benicewicz, L. S. Schadler, D. Acehan, A. Z. Panagiotopoulos, V. Pryamitsyn, V. Ganesan, J. Ilanvsky, P. Thiyagarajan, R. H. Colby, and J. F. Douglas, Nat. Mater. 8, 354 (2009).

${ }^{12}$ A. Chremos and A. Z. Panagiotopoulos, Phys. Rev. Lett. 107, 105503 (2011).

${ }^{13}$ M. P. Allen and D. J. Tildesley, Computer Simulations of Liquids (Oxford University Press, New York 1987), Chap. 8.

${ }^{14}$ H. C. Andersen, J. Chem. Phys. 72, 2384 (1980).

${ }^{15}$ M. G. Mazza, N. Giovambattista, F. W. Starr, and H. E. Stanley, Phys. Rev. Lett. 96, 2 (2006).

${ }^{16}$ R. A. Reis, F. C. Silva, R. Nobrega, J. V. Olivera, and F. W. Tavares, Fluid Phase Equilib. 221, 25 (2004).

${ }^{17}$ S.-H. Chong and W. Kob, Phys. Rev. Lett. 102, 1 (2009).

${ }^{18}$ A. Z. Akcasu, N. Corngold, and J. J. Duderstadt, Phys. Fluids 13, 2213 (1970).

${ }^{19}$ A. R. C. Baljon and M. O. Robbins, Comput. Theor. Polym. Sci. 9, 35 (1999).

${ }^{20}$ J. Rottler, S. Barsky, and M. O. Robbins, Phys. Rev. Lett. 89, 148304 (2002). 
${ }^{21}$ S. W. Sides, G. S. Grest, M. J. Stevens, and S. J. Plimpton, J. Polymer Sci., Part B: Polymer Phys. 42, 199 (2004).

${ }^{22}$ R. Edberg, D. J. Evans, and G. P. Morriss, J. Chem. Phys. 84, 6933 (1986).

${ }^{23}$ R. Edberg, D. J. Evans, and G. P. Morriss, J. Chem. Phys. 86, 4555 (1987).

${ }^{24}$ A. W. Lees and S. F. Edwards, J. Phys. C 5, 1921 (1972).

${ }^{25}$ L. M. Hood, D. J. Evans, and G. P. Morriss, Mol. Phys. 62, 419 (1987).

${ }^{26}$ J. F. Brady, J. Chem. Phys. 98, 3335 (1993).

${ }^{27}$ J. F. Brady, J. Chem. Phys. 99, 567 (1993).

${ }^{28}$ D. R. Foss and J. F. Brady, J. Fluid Mech. 407, 167 (2000).
${ }^{29}$ F. Rodriguez, C. Cohen, C. K. Ober, and L. A. Archer, Principles of Polymer Systems, 5th ed. (Hemishphere, New York, 2003).

${ }^{30} \mathrm{M}$. Doi and S. F. Edwards, Theory of Polymer Dynamics, 1st ed. (Oxford Science, Oxford, 1986).

${ }^{31}$ W. P. Krekelberg, T. Kumar, J. Mittal, J. R. Errington, and T. M. Truskett, Phys. Rev. E 80, 061205 (2008).

${ }^{32}$ M. J. Pond, J. R. Errington, and T. M. Truskett, J. Chem. Phys. 134, 081101 (2011).

${ }^{33}$ M. J. Pond, J. R. Errington, and T. M. Truskett, Soft Matter 7, 9859 (2011). 